\title{
An empirical comparison of techniques for the class imbalance problem in churn prediction
}

\author{
Bing Zhu ${ }^{\mathrm{a}, \mathrm{b}}$, Bart Baesens ${ }^{\mathrm{b}, \mathrm{c}, *}$, Seppe K.L.M. vanden Broucke ${ }^{\mathrm{b}}$ \\ ${ }^{a}$ Business School, Sichuan University, Chengdu 610064, P.R. China \\ ${ }^{b}$ Department of Decision Sciences and Information Management, KU Leuven, Leuven 3000, Belgium \\ ${ }^{c}$ School of Management, University of Southampton, Highfield Southampton SO17 1BJ, United Kingdom
}

\begin{abstract}
Class imbalance brings significant challenges to customer churn prediction. Many solutions have been developed to address this issue. In this paper, we comprehensively compare the performance of state-of-the-art techniques to deal with class imbalance in the context of churn prediction. A recently developed expected maximum profit criterion is used as one of the main performance measures to offer more insights from the perspective of cost-benefit. The experimental results show that the applied evaluation metric has a great impact on the performance of techniques. An in-depth exploration of reaction patterns to different measures is conducted by intra-family comparison within each solution group and global comparison among the representative techniques from different groups. The results also indicate there is much space to improve solutions' performance in terms of profit-based measure. Our study offers valuable insights for academics and professionals and it also provides a baseline to develop new methods for dealing with class imbalance in churn prediction.
\end{abstract}

Keywords:

Churn prediction, Class imbalance, Benchmark experiment, Expected maximum profit measure

\section{Introduction}

Customer churn prediction is an important issue in customer relationship management. In today's increasingly competitive business environment, customers can easily switch between competitors. Several studies have shown that acquiring a new customer is usually five to six times more expensive than retaining an existing customer [5, 14]. Meanwhile, long-term customers tend to be less sensitive to competitive marketing activities and produce higher profits $[22,61]$. Therefore, companies are shifting their focus from acquiring new customers to retaining their existing customer base. Accurate churn prediction helps companies to target the right customers to retain and it has hence been recognized as a marketing priority. Consequently, we have witnessed various applications of data mining techniques in this field $[26,66]$.

\footnotetext{
${ }^{*}$ Corresponding author. Tel.: +32 (0)16326884; fax: +32 (0)16326732

Email address: Bart.Baesens@kuleuven. be (Bart Baesens)
} 
Customer churn is a rare event in many industries, meaning that the number of churners is significantly outnumbered by non-churners. In the telecom sector, for example, the monthly churn rate is roughly $2 \%$. Thus customer churn prediction can be presented as a binary classification task with an imbalanced class distribution [2], where the churners belong to the minority class and non-churners belong to the majority class. The Class imbalance problem produces a great negative influence on standard classification learning algorithms. Most of them tend to bias toward to the majority class [54]. In extreme cases, they may classify all instances to the majority class, resulting in high overall accuracy but unacceptably low precision with respect to the minority class of interest. For instance, when a model is trained on a data set with $1 \%$ of instances from the minority class, a $99 \%$ accuracy rate can be achieved by simply classifying all instances as belonging to the majority class. Indeed, the issue of learning on imbalanced data sets is considered to be one of the ten challenging problems in data mining research as listed in [58].

In order to solve the problem of learning from imbalanced data sets, many solutions have been proposed in the past few years $[54,28]$. Most well-known proposed solutions roughly fall into three groups: data-level, algorithmlevel and ensemble solutions. Data-level solutions apply resampling as a preprocessing step to reduce the negative effect caused by class imbalance. Algorithm-level solutions aim to develop new algorithms or modify existing ones to bias learning towards the minority class. Ensemble solutions either modify the ensemble learning algorithms at the data-level to preprocess the data before the learning stage of base classifiers or embed a cost-sensitive framework in the ensemble learning process. Each type of solution has pros and cons, without a clear consensus on what makes for the best solution to address the problem of class imbalance in churn prediction.

In this paper, our aim is to experimentally compare state-of-the-art methods for dealing with class imbalance on real-world churn prediction data sets. Our contributions are twofold: first, we launch a large scale benchmark study of 21 techniques across 11 real churn data sets. So far, no such exhaustive benchmark comparison was performed in the context of churn prediction. Several techniques are assessed for the first time in this field. Second, we include a recently-developed profit-based measure called Expected Profit Measure (EMP) as one of the performance measures in the experiment. As pointed out by Raeder [47], the choice of evaluation metrics plays an important role in imbalanced learning. Traditionally, the area under the receiver operating curve (AUC) and top-decile lift are widely used in churn prediction [40]. However, both measures do not take the real costs and benefits brought by the churn model into account. Meanwhile, the EMP measure is developed from a cost-benefit perspective by Verbraken et al. [69]. It combines the outputs of churn prediction models with the profits brought by the retention campaign. Therefore, our study tries to explore the performance of techniques dealing with class imbalance by using the EMP measure together with the traditional AUC and top-decile lift measure. Experimental results show that the used evaluation metrics have a great impact on the performance of the techniques. We offer an in-depth exploration of the sensitivity of each technique with respect to each evaluation measure. We find that there is still much room to improve the techniques handling class imbalance in terms of the profit-based measure in churn prediction.

The remainder of this paper is organized as follows. In Section 2, we briefly review churn prediction research and describe performance measures used in this field. In Section 3, we discuss the solutions to class imbalance. Section 
4 presents the detailed experimental methodology and Section 5 analyzes the experimental results. Finally, Section 6 gives conclusions and directions for further research.

\section{Churn prediction}

\subsection{Churn prediction modeling}

Churn prediction aims at identifying potential churning customers based on past information and prior behavior and offering them some incentives to stay. Neslin et al. [45] suggested that prediction techniques have a huge impact on the returns of subsequent retention actions and that a small improvement in a churn prediction model can lead to a significant increase in profits. As a result, the optimization of churn prediction models has been explored extensively in the last few years.

The quality of churn prediction model is determined by both data and learning algorithm. Therefore, current studies focus on these two aspects to optimize the model performance models. From the aspect of learning algorithms, customer churn prediction involves binary classification and numerous classification algorithms from the research fields of machine learning and statistics have been adopted such as logistic regression [8], decision trees [41], neural networks [31], support vector machines [16], partial least squares(PLS) [46] and hazard models [36]. Due to the ease of use and interpretability, logistic regression and decision trees are commonly used by academics and practitioners [45]. However, more advanced models also demonstrated their predictive power with the increase of model complexity. For example, Keramati et al. [37] used decision trees, artificial neural networks, and k-nearest neighbor for predicting customer churn in a telecommunication company, where the results showed the superiority of artificial neural networks. Gordini and Veglio [25] developed a churn prediction model tailored for B2B e-commerce industry based on support vector machines (SVM). Their experimental results showed SVM outperforms logistic regression and neural networks.

All the works listed above implement the prediction model as a single model. Recently, researchers have also explored the usage of so-called ensemble or hybrid learning techniques in customer churn prediction, which have demonstrated substantial improvement over a single classification model. For example, stochastic gradient boosting, and bagging models have been used by Lemmens and Croux [40] to model churn for a wireless telecommunications company, which showed the two ensemble methods obtain substantial better results than logistic regression. Tsai and $\mathrm{Lu}[56]$ tested a hybrid data mining technique which combines back-propagation artificial neural networks (ANN) and self-organizing maps in telecom churn prediction, and found that the hybrid models outperform the single neural network baseline model in terms of prediction accuracy. Although various algorithms have been applied, there appears no single dominating approach which is useful in every churn-related context. For an extensive literature review on customer churn prediction techniques, we refer the interested reader to $[26,66]$.

The second research stream puts the emphasis on the data used to build the churn model. Some researchers tried to investigate the usefulness and representation of certain types of data information. For instance, Zhang et al. [62] 
investigated the effect of interpersonal influence information on the accuracy of churn prediction, and combined it with customers' personalized characters to produce a new prediction model. Verbeke et al. [68] used a relational learning algorithm to explore social network information within a customer churn prediction setting. Chen et al. [12] integrated static customer data and longitudinal behavioral data to improve the performance of prediction models. Other researchers investigated the impact of data pre-processing on the model predictive accuracy. Huang et al. [32] proposed a new multiobjective feature selection approach based on a non-dominated genetic algorithm (NSGA) and proved its success in churn prediction. Coussement et al. [17] examined data-preparation alternatives to enhance the prediction performance in a churn prediction modeling context. Their study showed the data-preparation technique actually affects prediction performance. All these data-oriented studies indicate that by careful checking the data characteristics and developing corresponding techniques the churn prediction model can significantly improve. However, the research in this field is still under-developed. The research in this paper falls into the second stream.

\subsection{Evaluation metrics in churn prediction}

Evaluating model performance is a key step in successful churn prediction modeling. Traditionally, AUC and top-decile lift are used as the main performance measures.

Receiver operating characteristic (ROC) curve analysis is one of the popular approaches that can be used to measure the performance of classifiers on imbalanced data sets. ROC analysis has its origin in signal detection theory as a method to choose a threshold or an operating point for the receiver to detect the presence or absence of a signal. Bradley [7] introduced ROC analysis to the machine learning field in 1997. The ROC graph plots true positive rates versus false positive rates. Classifiers can be selected based on their tradeoffs between true positives and false positives. The AUC can be expressed as follows:

$$
A U C=\int_{0}^{1} \operatorname{TPR}(t) d F P R(t)
$$

where $t$ is the threshold, TPR is true positive rate and FPR is the false positive rate. Rather than visually comparing curves, the area under the ROC curve metric (AUC) aggregates the performance of a classification model into a single number, which makes it easier to compare the overall performance of multiple classification models. A random classifier has an AUC of 0.5 and a perfect classifier possesses an AUC equal to 1.

Top-decile lift is another widely used performance measure in churn prediction. To calculate top-decile lift, customer instances are first sorted based on the churn propensity scores obtained by the prediction model in a descending order. Then, the top-decile lift is computed as the ratio of the percentage of correctly classified churners (the minority class) in the top $10 \%$ ranked cases $\left(\beta_{10 \%}\right)$ and the percentage of actual churners in the entire data set as follows:

$$
\text { top-decile } \quad \text { lift }=\beta_{10 \%} / \beta_{0}
$$

For instance, a top-decile lift value of 2 indicates that the classifier identifies twice as many churners in the top $10 \%$ ranked customer group as a random classifier would do. 


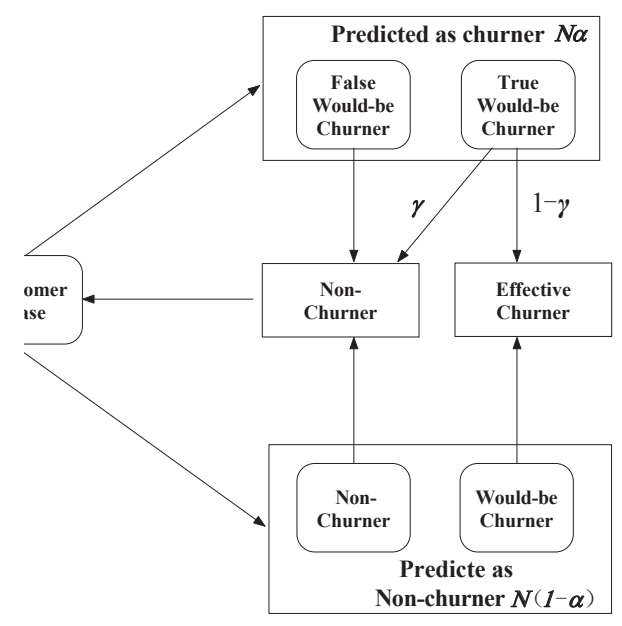

Figure 1: The dynamic process of customer churn and retention

Although AUC and top-decile lift are two widely used performance measures in churn prediction, both measures do not take the real cost/benefit brought by churn models and consequent retention campaigns into account. To overcome the ignorance of the cost-benefit factor, profit-based measures were recently proposed by Verbraken et al. [69] and Verbeke et al. [67]. Before giving the description of these measures, let us outline the dynamical process of customer churn and retention programs as presented in Fig. 1. As the figure shows, after building a churn prediction model, the firm will first apply the model to its current customer base. Then, a fraction $\alpha$ of customers with the highest churn propensities are targeted and offered some incentives. There are true and false would-be churners within the target customers. In the true would-be churner group, a fraction $\gamma$ of customers will accept the offer and stay active, whereas the remaining fraction $1-\gamma$ will still defect. In the false would-be churner group, the assumption here is that all customers will accept the incentives and that they do not churn because they had no intention to leave. Meanwhile, in the $1-\alpha$ fraction (the non-target group), all would-be churners will leave whereas non-would-be churners will stay. We can see that the profit of launching a retention program is influenced by customers' churn propensities in the target fraction, their probabilities of accepting incentive offers, the cost of the incentive offer, customer lifetime values and so on. Neslin et al. [45] established the following expression to get the total profit for a retention campaign:

$$
P=N \alpha[\beta \gamma(C L V-c-\delta)+\beta(1-\gamma)(-c)+(1-\beta)(-c-\delta)]-A
$$

where $P$ is the profit of the retention campaign, $N$ is the number of customers in the current customer base, $\alpha$ is the fraction of the target customers in the retention campaign, $\beta$ is the portion of churners within the targeted customers, $\gamma$ is the probability of would-be churners accepting the offer and staying in the company, $C L V$ is the average customer lifetime value of retained customers, $c$ is the cost of the incentive when a customer accepts the offer, $\delta$ is the cost of contacting a customer to offer the incentive, and $A$ is the fixed administrative cost of running a retention program. 
The first term in the bracket of Equation (3) reflects the revenue contributed by the retained potential churners. The second term presents the loss coming from the identified potential customers who do not accept the offer and finally defect. The last term in the bracket is the loss of sending incentives to the customers who are actually non-churners. This equation can be also rewritten as follows:

$$
P=N \alpha\left[(\gamma C L V+\delta(1-\gamma)) \beta_{0} \lambda-\delta-c\right]
$$

where $\beta_{0}$ is churn rate in the whole customer base, $\lambda$ is the lift corresponding to the target fraction $\alpha$. Based on equation (4), Verbeke et al. [67] presented the maximum profit (MP) criterion which is defined as follows:

$$
M P=\max _{\alpha}\left(N \alpha\left[(\gamma C L V+\delta(1-\gamma)) \beta_{0} \lambda-\delta-c\right]\right)
$$

By selecting the optimal target size $N \alpha$, the MP measure will give the maximum profit that a retention campaign can achieve given the churn prediction model. In the MP measure, all parameters except target size are supposed to be known. In practice, customer lifetime value $C L V$, the cost of the incentive $\delta$ and the contacting cost $\phi$ can be estimated with sufficient reliability. However, estimating the probability of a churner accepting the retention offer is not a trivial task. Therefore, Verbraken et al. [69] adopted a probability distribution for $\gamma$ and presented a probabilistic profit-based performance measure, i.e. the expected maximum profit measure (EMP) for a churn model as follows:

$$
E M P=\int_{\gamma} P_{c}(T(\gamma) ; C L V, \delta, \phi) \cdot h(\gamma) d \gamma
$$

where $h(\gamma)$ is the probability density function for $\gamma$. Given a $\gamma$ value, $T(\gamma)$ is the optimal threshold and $P_{c}(T(\gamma) ; C L V, \delta, \phi)$ is the maximum profit calculated as in equation (5). $\gamma$ is assumed to follow a Beta distributed random variable $B(\alpha, \beta)$ with two parameters $\alpha$ and $\beta$. More details about the empirical calculation of the EMP measure can be found in [69]. Both MP and EMP measures have been successfully used in churn prediction [67,69] and it has been shown that using the two profit-based measures to select churn prediction models will provide a significant increase in profits compared to AUC and top-decile lift. By taking into account the uncertainty of the classification costs and benefits, the EMP measure provides a more general form to measure the expected profits produced by churn models and we will use it as one of the main measures in our experimental study.

\section{Class imbalance}

Class imbalance is an importance characteristic of many data sets used for churn prediction modeling. Most standard classification algorithms frequently exhibit a bias toward the majority class, so class imbalance often hinders the performance of standard classifiers, especially in terms of the minority class of interest. Weiss [72] drew up six types of problems brought on by imbalanced data sets. More details about how class imbalance can influence different classification algorithms can be found in [54]. A great variety of solutions have been proposed to address this issue, 
which can be roughly divided into three categories: data-level, algorithm-level and ensemble solutions. We will give a brief description for each type of solution in the following subsections.

\subsection{Data-level solution}

Data-level solutions try to re-balance the class distribution by resampling the original, unbalanced data set. Datalevel solutions consist of many different forms of resampling techniques. Random sampling techniques offer the simplest approaches, which include random oversampling (ROS) and random undersampling (RUS). ROS tries to create a superset of the original data set by randomly replicating the minority instances from the existing data set. RUS aims to balance the class distribution through random elimination of majority class examples. Both techniques are easy to use and understand, though RUS can discard potentially useful data instances whereas ROS can increase the likelihood of overfitting by making exact copies of existing instances. In order to deal with these drawbacks, some intelligent sampling methods have been proposed, of which synthetic minority oversampling technique (SMOTE) [10] is probably the most popular one. SMOTE first randomly selects several nearest neighbors of a minority class instance and produces new instances based on linear interpolations between the original examples and the randomly selected nearest neighbors. Although SMOTE is well acknowledged in the academic community, it still suffers from some drawbacks. For instance, SMOTE generates the same number of synthetic minority instances for each original minority example and does not take neighboring examples belonging to the majority class into consideration, which can result in an increase of overlap between the classes. To overcome the shortcomings of SMOTE, some variants have been developed. Adaptive synthetic sampling (ADASYN) uses the density distribution to decide on the number of synthetic samples that need to be generated for each minority example [27]. ADASYN first finds the nearest neighbors for each minority instance and then counts the number of majority examples in these neighbor instances to calculate a ratio. The number of instances generated for each instance is then decided by this ratio. Recently, a new intelligent sampling method, called majority weighted minority oversampling technique (MWMOTE) was also presented [3]. MWMOTE first identifies the hard-to-learn informative minority class samples and assigns them weights according to the distance from the nearest majority class samples. It then generates synthetic samples from the weighted informative minority samples using a clustering approach.

Some intelligent methods for undersampling have also been proposed. Kubat and Matwin [38] proposed the one-sided selection (OSS) technique, which selectively removes the majority instances that either are redundant or borderline majority examples. The cluster-based undersampling algorithm (CLUS) presented by Yen et al. [60] organizes training data into groups with homogeneous characteristics and then downsizes the number of majority class samples in each cluster.

There are also several hybrid sampling techniques. Some of them combine oversampling with data cleaning techniques to reduce the overlapping introduced by oversampling methods. Typical examples are SMOTE-Tomek and SMOTE-ENN [4]. SMOTE-Tomek finds pairs of minimally distanced nearest neighbors of opposite classes. When two samples form a Tomek link, either one of them is noisy or both samples are borderline instances. SMOTE-Tomek 
deletes Tomek links after SMOTE sampling so that all minimally distanced nearest neighbor pairs are in the same class. SMOTE-ENN is similar to SMOTE-Tomek, but it uses Wilson's edited nearest neighbor (ENN) rule to remove instances after SMOTE sampling. That is, any example that is misclassified by its three nearest neighbors is removed from the data set. Apart from the usage of data cleaning techniques, some researchers presented hybrid sampling methods that apply computational intelligence technologies to identify useful instances. For instance, García and Herrera [21] proposed a method called evolutionary under-sampling, which uses evolutionary algorithms to select prototype instances.

\subsection{Algorithm-level solution}

This category tries to strengthen the learning of classification algorithms with regards to the minority class. The most popular branch in this group is cost-sensitive learning. Cost-sensitive methods assign different misclassification costs for different classes, generally a high cost for the minority class and a low cost for the majority class. The costsensitive learning process then tries to minimize the total cost of misclassification. So far, many cost-sensitive learning methods have been proposed to deal with class imbalance. For instance, Ting presented an instance-weighting method to induce cost-sensitive trees [57]. Veropoulos et al. proposed a biased support vector machine algorithm by setting different costs to the majority and minority class in the objective function [70].Zhou and Liu [64] have proposed a cost-sensitive neural network based on threshold adjustment to address the class imbalance problem.

Other algorithm-level solutions include one-class learning and active learning. One-class learning aims to recognize instances of a concept by using only a single class of examples rather than differentiating between instances of both classes. One representative type of one-class learners are one-class SVMs [48]. Active learning methods were originally developed to solve problems related to unlabeled training data. Recently, it has found increasing usage in imbalanced learning applications. For example, Ertekin et al. proposed an SVM-based active learning method which queries a small pool of data at each iterative step of active learning instead of using the entire data set [19]. In this paper, we choose the most popular cost-sensitive learning as the representative technique of this group.

\subsection{Ensemble solution}

Ensemble solutions have become popular in recent years. A good review of ensemble solution for learning with imbalanced classes can be found in [24]. Ensemble learning tries to improve the performance of single classifiers by inducing several classifiers and combining them to obtain a new, better performing classifier. To address the class imbalance issue, ensemble solutions combine ensemble learning with the above mentioned data or algorithmic level approaches. Most ensemble solutions are based on known strategies such as bagging, boosting, random forests and their hybrids.

1. Bagging-based ensemble: Bagging-based ensembles integrate bagging with data sampling techniques. They collect the training samples and use different sampling methods to generate training sets and combine classifiers trained for each training set. For example, Underbagging and Overbagging [71] use random undersampling or 
oversampling so that the class distribution is balanced when building each model in the ensemble. Roughly Balance Bagging [29] is also based on undersampling, but the size of the majority examples is determined probabilistically according to a negative binomial distribution, whereas the number of minority examples is always the same. In SMOTEBagging [71], each training data set is composed of a bootstrap sample taken from the majority class, and a sample of the minority class created through a combination of oversampling and SMOTE.

2. Boosting-based ensemble: Boosting-based ensembles include techniques that embed data preprocessing or cost-sensitive learning in a boosting procedure. Cost-sensitive boosting keeps the general learning framework of boosting algorithms, but introduces cost items into the weight update formula. Well-known cost-sensitive boosting techniques are AdaC1, AdaC2, AdaC3, CSB1 and CSB2 [53]. These methods usually differ in the way that they modify the weight update rule. Data-preprocessing-based boosting exploits sampling to bias the data distribution towards the minority class before the classifier generation step. For example, Chawla et al. proposed an approach called SMOTEBoost by the combination of SMOTE sampling and a boosting procedure [11], in which SMOTE sampling is used before computing the new example weights in each iteration. Seiffert et al. [51] presented a different ensemble method named RUSBoost, which removes instances from the majority class by random undersampling in each iteration of boosting.

3. Random forest-based ensemble: Chen et al. [13] proposed the balanced random forest (BRF) and weighted random forest (WRF) models to learn from imbalanced data. The idea of BRF is to combine undersampling with a random forest approach. For each repetition, the same number of instances from the minority class and the majority class are randomly drawn with replacement, respectively. WRF follows the idea of cost-sensitive learning, where a heavier penalty is placed on the node-splitting condition and terminal-node class weights for misclassifying the minority class.

4. Hybrid ensemble: Representative techniques in this category include EasyEnsemble and BalanceCascade [42], which are hybrid approaches that combine bagging and boosting. More specifically, EasyEnsemble uses bagging as the main ensemble learning method. Instead of training a single classifier in each bag, Adaboost is used to train on a balanced dataset formed by random undersampling. BalanceCascade works in a sequentially supervised manner. In each bagging iteration after learning the AdaBoost classifier, the majority class examples that are correctly classified by the current Adaboost classifier are permanently removed from the data set.

Some other ensemble solutions based on ensemble selection or ensemble pruning are also explored. Two typical examples are the ordering-based ensemble pruning proposed by Galar et al. [23] and genetic-programming-based ensemble selection by Bhowan [6], which refine the ensemble by choosing the best ensemble members. However, the above mentioned four types of ensemble solutions are still considered the state-of-the art methods

\subsection{Summary and comments}


Although various solutions have been proposed, each type of solution has pros and cons. Data-level approaches are simple and easy to use, but shortcomings are also obvious. The sampling methods alter original data class distribution of imbalanced data which may lead to some unexpected mistakes [55]. Moreover, the optimal class distribution of the training data is usually unknown [53]. The algorithm-level solutions are specific to a certain classification algorithm and are hence often sensitive to the context in which they are applied. To develop an algorithm level solution, one needs to possess extensive knowledge about both the learning algorithm and application domain. Ensemble solutions embed either data-level or algorithm-level solutions in the ensemble learning framework. Therefore, they will inherit the drawbacks of the two solutions. Moreover, ensemble solutions also bring extra model complexity and computational cost.

In order to figure out better solutions for dealing with class imbalance, many comparative studies have been done. Van Hulse et al. [65] presented a comprehensive experiment with eight sampling methods. It was found that random sampling methods perform better than intelligent sampling methods like SMOTE. García et al. [20] investigated the influence of both imbalance ratio and classifier on several resampling strategies to deal with imbalanced data sets. Experiments showed that oversampling consistently outperforms undersampling when data sets are strongly imbalanced, whereas there are no significant differences on data sets with a low imbalance. Khoshgoftaar et al. [39] compared boosting with bagging techniques in the context of noisy and imbalanced data. The experiments showed that bagging techniques generally outperform boosting in noisy data environments. Galar et al. [24] developed a thorough empirical comparison by the consideration of a wide range of ensembles, from simple modifications of bagging and boosting to cost-sensitive or hybrid approaches. Their main conclusions state that SMOTEbagging, RUBoost and UnderBagging give the best AUC results. López et al. [43] analyzed the performance of sampling techniques against cost-sensitive learning in the framework of imbalanced learning. The results showed that there are no differences among them. López [44] et al. carried out an experimental study to contrast sampling, cost-sensitive learning and ensemble techniques. The results highlighted the dominance of ensemble approaches UnderBagging and SMOTEBagging when C4.5 and k-NN are used as weak classifiers while the best results are achieved by SMOTE sampling and cost-sensitive learning when SVM is considered. Other comparative studies can also be found in [49, 50]. To summarize the above comparative studies, we find that conclusions about the performance of different solutions do not reach a clear consensus.

In churn prediction, the solutions to deal with class imbalance do not go beyond the scope of data-level, algorithmlevel and ensemble solution. For example, Chen et al. [12] used random undersampling as a data-preprocessing strategy to churn prediction. SMOTE sampling is used by Ali and Aritürk [1] in their dynamic modeling framework. Idris et al. [33] investigated the significance of particle swarm optimization (PSO) based undersampling method to handle the imbalance data distribution. Xie et al. [76] proposed the improved balanced random forests approach, which combines balanced random forests and weighted random forests. Xiao [75] proposed a method that handles class imbalance by combining ensemble learning with cost-sensitive learning. 


\section{Experimental framework}

Since model performance on imbalanced data sets is influenced by many factors, we argue that it is not feasible to determine which technique is best suited in any general context. Therefore, we narrow our contribution and scope to the churn prediction domain. Burez and Van den Poel [9] have performed some pioneering studies to compare different solutions for churn prediction. But only two sampling techniques and one cost-sensitive ensemble learning technique were considered. Besides, they only consider AUC like most previous studies.

In this paper, we will conduct a more comprehensive experimental study and present an in-depth exploration with the domain-specific profit-based measure together with the commonly used AUC measure. Different from our previous research in [63], which only compares the data-level solutions and focuses on the MP measure, we have also included the algorithm-level and ensemble solutions and consider the more general EMP measure. To summarize, we aim at answering the following research questions:

RQ1 Do different evaluation metrics have similar influence on the performance of different solutions?

RQ2 Does each solution truly improve the performance in comparison with the original technique without considering class imbalance?

RQ3 What is the best technique given an evaluation measure?

In this section, we present the framework that will be used to carry out the experiments. First, we provide details of the real-world customer data sets used in Subsection 4.1. Then, we give a brief summary of all the techniques used in the experiment as well as other experimental settings in Subsection 4.2. Finally, Subsection 4.3 presents the statistical tests used in the experimental analysis.

\subsection{Data sets}

The eleven real-world data sets used in the experiments stem from the telecommunication industry. Table 4.1 summarizes the main characteristics of the data sets, where each column presents name, abbreviation (Abbr.), data source, region, number of observation (\#Obs.), attributes (\#Att.) and churn rate. As Table 1 shows, the churn rates range from $1.8 \%$ to $14.5 \%$, and most of them are highly imbalanced.

Two steps are performed for data preparation before proceeding with model construction. The first step involves dealing with missing values. In case of categorical variables, the missing values are encoded as a new level. For continuous variables, if more than $50 \%$ of the values are missing, the variable is removed. Otherwise, median imputation is used to avoid the influence of extreme values. In order to avoid the "curse of dimensionality" problem, a feature selection procedure was conducted as the second step for data preparation. More specifically, a feature selection approach based on the Fisher score is used on five data sets: Chile, Duke1, Duke2, KDD and Tele1. The following formula is applied to calculate the Fisher score for each variable: 


$$
\text { Fisher Score }=\frac{\left|\bar{x}_{c}-\bar{x}_{n c}\right|}{\sqrt{s_{c}^{2}+s_{n c}^{2}}}
$$

where $\bar{x}_{c}$ and $\bar{x}_{n c}$ are the mean value, and $s_{c}^{2}$ and $s_{N c}^{2}$ are the variances of variable for the churners and the non-churners, respectively. Features with highest Fisher scores on the five above mentioned data sets are retained and the number of variables in these data sets is reduced to 30 as including more variables does not necessarily increase performance.

Table 1: Summary of churn data sets used in the experiments

\begin{tabular}{|c|c|c|c|c|c|c|}
\hline Dataset & Abbr. & Source & Region & \#Obs. & \#Att. & Churn Rate (\%) \\
\hline Chile & Chile & Operator & South American & 5300 & 41 & 5.66 \\
\hline Duke_current & Duke1 & Duke & North American & 51306 & 173 & 1.80 \\
\hline Duke_future & Duke2 & Duke & North American & 100462 & 173 & 1.80 \\
\hline KDDcup & KDDcup & KDD CUP 2009 & Europe & 50000 & 231 & 7.34 \\
\hline Korean1 & $\mathrm{K} 1$ & Operator & East Asia & 2019 & 10 & 3.96 \\
\hline Korean2 & $\mathrm{K} 2$ & Operator & East Asia & 2941 & 14 & 4.42 \\
\hline Korean3 & K3 & Operator & East Asia & 5990 & 36 & 4.34 \\
\hline Korean 4 & $\mathrm{~K} 4$ & Operator & East Asia & 2183 & 9 & 4.58 \\
\hline Korean5 & K5 & Operator & East Asia & 26224 & 11 & 4.19 \\
\hline Tele1 & Tele1 & Operator & Europe & 4350 & 87 & 8.05 \\
\hline UCI & UCI & UCI ML repository & - & 3333 & 19 & 14.5 \\
\hline
\end{tabular}

\subsection{Experimental settings}

A five times two-fold cross-validation strategy was applied in our study as follows: each original data set was randomly split into two equally-sized parts. First, one part was used as training data to build models, while the other part acted as test data to calculate the performance. Then, the two parts switched their roles. This process was repeated five times to get average values of each performance measure. We selected AUC, top-decile lift and expected maximum profit (EMP) to measure model performance. To calculate the EMP measure, the parameters $C L V, \delta$, and $c$ in Equation (6) were set to be 200, 10 , and 1, respectively. The two parameter in $B(\alpha, \beta)$ were set to be 6 and 14. The parameter settings were based on previous scientific literature [45, 35, 69] and discussion with data scientists in the telecommunication industry. Twenty-one techniques were chosen from the data-level, algorithm-level and ensemble solutions. All the selected methods have been listed in Table 2 together with their short description and parameter settings. Most parameter values were selected according to the recommendation of the corresponding authors of each algorithm. As far as we know, many solutions are applied here for the first time in a churn prediction task, like SMOTE-ENN, UnderBaging and EasyEnsemble. The experiments were conducted using the open-source R language. 
Table 2: Techniques used in the experimental study

\begin{tabular}{|c|c|c|}
\hline Methods & description & parameters \\
\hline \multicolumn{3}{|c|}{ (a) Sampling solution } \\
\hline AYDYSN & Adaptive synthetic sampling [27] & Number of nearest neighbors $K=5$ \\
\hline CLUS & Cluster-based undersampling algorithm [60] & Number of clusters $K=3$ \\
\hline MWMOTE & $\begin{array}{l}\text { Majority weighted minority oversampling technique } \\
\text { [3] }\end{array}$ & $\begin{array}{lcl}k 1=5, & k 2=3, & k 3=\left|S_{\min }\right| / 2 \\
C_{p}=3, & C_{f}(t h)=5, & C M A X=2\end{array}$ \\
\hline ROS & Random oversampling & - \\
\hline RUS & Random undersampliing & - \\
\hline SMOTE & Synthetic minority oversampling [10] & Number of nearest neighbors $K=5$ \\
\hline SMOTE-ENN & SMOTE sampling with ENN cleaning [4] & Number of nearest neighbors in SMOTE $K=5$ \\
\hline SMOTE-Tomek & SMOTE sampling with Tomek-link cleaning [4] & Number of nearest neighbors in SMOTE $K=5$ \\
\hline \multicolumn{3}{|c|}{ (b) Cost-sensitive solution } \\
\hline CS-Tree & Cost-sensitive trees based on in instance-weighting [57] & $C_{\text {non-churner }}=11, C_{\text {churner }}=56$ \\
\hline CS-SVM & Cost-sensitive support vector machine [70] & $C_{\text {non-churner }}=11, C_{\text {churner }}=56$ \\
\hline \multicolumn{3}{|c|}{ (c) Ensemble solution } \\
\hline Bagging & Bagging & Number of bags $N=40$ \\
\hline OverBagging & OverBagging [71] & Number of bags $N=40$ \\
\hline RBBagging & Roughly balanced bagging [29] & Number of bags $N=40$ \\
\hline SMOTEBagging & SMOTEBagging[71] & $\begin{array}{l}\text { Number of bags } N=40 \\
\text { Number of nearest neighbors in SMOTE } K=5\end{array}$ \\
\hline UnderBagging & UnderBagging [71] & Number of bags $N=40$ \\
\hline AdaBoost & AdaBoost & Number of iterations $T=40$ \\
\hline AdaC2 & AdaC2 [53] & $\begin{array}{l}\text { Number of iterations } T=40 \\
C_{n o n-\text { churner }}=11, C_{\text {churner }}=56\end{array}$ \\
\hline RUSBoost & RUSBoost [51] & Number of iterations $T=40$ \\
\hline SMOTEBoost & SMOTEBoost [11] & Number of iterations $T=40$ \\
\hline $\mathrm{RF}$ & Random forest & Number of trees $N=40$ \\
\hline BRF & Balanced random forest [13] & Number of trees $N=40$ \\
\hline WRF & weighted random forest [13] & $\begin{array}{l}\text { Number of trees } N=40 \\
C_{\text {non-churner }}=11, C_{\text {churner }}=56\end{array}$ \\
\hline EasyEnsemble & EasyEnsemble[42] & $\begin{array}{l}\text { Number of subsets } S=4 \\
\text { Number of iterations in AdaBoost } T=10\end{array}$ \\
\hline BalanceCascade & BalanceCascade[42] & $\begin{array}{l}\text { Number of subsets } S=4 \\
\text { Number of iterations in AdaBoost } T=10\end{array}$ \\
\hline
\end{tabular}


Eight sampling methods were chosen as the representatives of data-level solutions. Sampling rate is an important parameter for all sampling methods. Our previous research has found that roughly balanced strategy results in universal good performance, in which the final class distributions were set to 1:3 (minority class vs majority class) [63]. Experimental results of sampling solutions were based on that configuration. For SMOTE-ENN and SMOTE-Tomek, one cannot control the exact class distributions, so we apply the same parameter setting as the one that lets SMOTE reach less balanced class ratios (1:3). For CLUS sampling, we have experimented with several values for the number of clusters $K \in\{3,5,7\}$ with similar results, so that the one requiring the minimum computational cost $K=3$ was selected. To investigate the impact of sampling methods, the following two classifiers were used as baselines:

1. C4.5 decision tree: We have set the confidence level to 0.25 , the minimum number of instances per leaf was set to 2 and pruning was used to obtain the final tree.

2. Support vector machine (SVM): With a radial basis function kernel (RBF), with heuristic search of the optimal kernel width $\sigma$ and the regularization term set to 1.0 .

For algorithm-level solutions, cost-sensitive learning methods were selected as the representatives. Two costsensitive learning methods were used in the experiments, the instance-weighting based cost-sensitive tree (CS-Tree) proposed by Ting [57] and the cost-sensitive support vector machine (CS-SVM) proposed by Veropoulos et al. [70]. The following costs were applied: misclassifying a non-churner as a churner leads to an average loss equal to the incentive offer value and the contact cost. Meanwhile, misclassifying a churner as a non-churner leads to an average loss equal to the expected net customer value minus contact cost. So, we set misclassification costs for non-churners $C_{n o n-\text { churner }}=\delta+c=11$ and churners as $C_{c h u r n}=E(\gamma(C L V-\delta)-c)=56$. Other parameters were kept to use the same settings as in the baseline classifiers.

Four types of ensemble solutions were considered in our study: bagging-based, boosting-based, random-forestbased and hybrid ensemble. Eleven techniques were considered in the experiments and we also included the original Bagging, AdaBoost and random forest algorithm to make the comparison. In all ensemble techniques, C4.5 decision tree was used as a base classifier, since most previous ensemble methodologies were proposed in combination with C4.5, with the settings kept equal to those as used in the base classifiers. We set the number of bags in bagging-based ensembles, the number of iterations in boosting-based ensembles and the number of trees in random-forest-based ensembles to be 40. For hybrid ensemble methods EasyEnsemble and BalanceCascade, 4 bags and 10 iterations were considered. These settings were consistent with previous research [42, 24] and our experiments also show that including more classifiers does not achieve better results. As noted above, there is a wide variety of cost-sensitive boosting techniques. Previous empirical studies showed that the AdaC2 algorithm stands out with respect to the others [53]. To avoid the repetition of similar experiments, we only empirically studied this algorithm from the set of cost-sensitive boosting techniques. In WRF and AdaC2, the misclassification costs were set to be the same as cost-sensitive solutions. 


\subsection{Statistical tests}

Following the recommendation of Demšar [18], two types of statistical tests were considered for pairwise comparison and multiple comparison, respectively. When only two algorithms were compared, a Wilcoxon signed-rank test was used [74]. For multiple comparisons, the Friedman test with Iman-Davenport extension was applied [34]. Iman-Davenport is based on the average ranks of the algorithms over multiple data sets. To get the average ranks, all algorithms were sorted from best to worst on each data set, where the best method obtained rank 1 , the second received rank 2 and so on. Then the ranks were averaged across all data sets. Given $N$ data sets, $k$ algorithms and average ranks $R_{i}, i=1, \ldots, k$, the Iman-Davenport test calculates a $F$-distribution statistic with $k-1$ and $(k-1)(N-1)$ degrees of freedom to validate the null hypothesis as follows:

$$
F_{F}=\frac{(N-1) \chi_{F}^{2}}{N(k-1)-\chi_{F}^{2}}
$$

where

$$
\chi_{F}^{2}=\frac{12 N}{k(k+1)}\left[\sum_{j} R_{i}^{2}-\frac{k(k+1)^{2}}{4}\right]
$$

If the null hypothesis that all methods have equivalent performance was rejected, a further post-hoc test should be applied to point out where the differences lay. In our study, we applied Holm's post-hoc test [30] to do so, as it can appropriately control the family-wise error rate (FWER) and hence is more powerful than Bonferroni-Dunn's test in a rigorous comparison [18]. Holm's test first selects the algorithm with the lowest mean rank as a control method, and then uses a step-down procedure to compare the control method with the other ones. The Holm's test utilizes the $Z$ statistic as follows:

$$
Z=\frac{\left(R_{i}-R_{j}\right)}{\sqrt{k(k+1) /(6 * N)}}
$$

where $R_{i}$ and $R_{j}$ are the average ranks of methods $i$ and $j$. After using the $Z$ statistic to find the corresponding probability from the normal distribution table, Holm's test orders $p$-values by $p_{1} \leq p_{2} \leq p_{3} \ldots \leq p_{k-1}$. Starting from the most significant $p$ value, Holm's procedure compares each $p_{i}$ with $\alpha /(k-i)(i=1,2, \ldots, k-1)$, where $\alpha$ is the given significance level. If $p_{1}$ is below $\alpha /(k-1)$, the corresponding hypothesis is rejected and we are allowed to compare $p_{2}$ with $\alpha /(k-2)$. If the second hypothesis is rejected, the test proceeds with the third, and so on. As soon as a certain null hypothesis cannot be rejected, all the remaining hypotheses are retained as well. In all statistical tests, we fixed the significance level $\alpha=0.05$

\section{Experimental results}

In this section, we offer the analysis of the experimental results. Interested readers can refer to the full experimental results in the Appendix. To answer the three research question in Section 4, we divide this section into five subsections. In the first three subsections, we will do intra-family analysis to investigate the results within each group of solution, separately. In the fourth subsection, we will perform a complete global comparison among the representatives coming from each family to find better performing techniques. In each subsection, we will explore behavior 
patterns of different techniques with respect to each evaluation measure. Finally, the fifth subsection summarizes the main findings from the experimental results.

\subsection{Comparison of sampling methods}

We start our investigation by comparing different sampling solutions. Table 3 shows the performance of different sampling techniques in combination with two baseline classifiers. Apart from the sampling methods, we also include a non-sampling strategy in the experiments to analyze whether the use of sampling is beneficial, which is denoted as "none". Each entry in the table gives the mean value of the three metrics as well mean ranks over eleven data sets and the last row shows the $p$-values of the Iman-Davenport test. The best rank in each column is in bold and the ranks that are significantly worse than the best one at the 5\% significance level are underlined based on Holm's post-hoc test. The results show that sampling methods indeed exhibit different behavior with different evaluation measures, and that this is interrelated with the classifier used.

Table 3: Experimental performance of sampling techniques

\begin{tabular}{|c|c|c|c|c|c|c|}
\hline \multirow{2}{*}{ Sampling } & \multicolumn{3}{|c|}{$\mathrm{C} 4.5$} & \multicolumn{3}{|c|}{ SVM } \\
\hline & AUC (Rank) & Top-decile lift (Rank) & EMP (Rank) & AUC (Rank) & Top-decile lift (Rank) & EMP (Rank) \\
\hline None & $0.6557(\underline{5.8182})$ & $4.5330(\mathbf{2 . 7 2 7 3})$ & 1.0359 (4.9091) & $0.6756(\underline{8.6364})$ & $2.7039(\underline{7.9091})$ & $0.7233(6.0000)$ \\
\hline ADASYN & $0.6899(4.4091)$ & $3.8553(5.6818)$ & $1.0542(4.4545)$ & $0.7264(4.8182)$ & $3.3536(4.5000)$ & $0.8858(5.0000)$ \\
\hline CLUS & 0.7255 (3.0909) & $3.6925(\underline{6.3636})$ & $0.9809(5.9091)$ & $0.7265(5.9091)$ & $3.2224(5.9545)$ & $0.8407(6.2727)$ \\
\hline MWMOTE & $0.6715(\underline{6.5455})$ & $3.7751(\underline{6.0000})$ & $1.0233(6.2727)$ & $0.7279(4.1818)$ & $3.3799(4.4091)$ & $0.8898(5.3636)$ \\
\hline ROS & $0.6464(\underline{8.1818})$ & $4.2573(5.6364)$ & $0.9958(6.5455)$ & 0.7326 (3.3636) & $3.4144(4.1818)$ & $0.8918(4.7273)$ \\
\hline RUS & $0.7322(\mathbf{2 . 5 4 5 5})$ & 3.8315 (4.5909) & 1.0590 (3.9091) & $0.7270(5.0909)$ & $3.3110(4.9091)$ & 0.8712 (3.9091) \\
\hline SMOTE & $0.6909(4.0455)$ & $3.8766(4.5000)$ & 1.0688 (4.0909) & $0.7269(5.1818)$ & $3.3840(4.9545)$ & $0.8928(4.9091)$ \\
\hline SMOTE-ENN & $0.6923(5.5455)$ & $3.9875(4.7273)$ & 1.0707 (4.6364) & $0.7335(3.5455)$ & $3.3831(4.2727)$ & $0.8943(4.6364)$ \\
\hline SMOTE-TL & $0.6885(4.8182)$ & $3.8906(4.7727)$ & $1.0668(4.2727)$ & $0.7297(4.2727)$ & 3.4136 (3.9091) & $0.8959(4.1818)$ \\
\hline Iman-Davenport & $p<0.0001$ & $p=0.0760$ & $p=0.0954$ & $p<0.0001$ & $p=0.0136$ & $p=0.5414$ \\
\hline
\end{tabular}

With respect to $\mathrm{C} 4.5$, the effectiveness of sampling methods depends on the measures as shown in Table 3 . In terms of AUC and EMP measure, most sampling methods have a positive impact on the results and RUS has the best results for both measures. On the contrary, the non-sampling strategy comes first when using top-decile lift, which means sampling techniques have a negative impact. According to Holm's test, RUS is significantly better than the nonsampling strategy and the other two sampling methods at the 5\% significance level with regards to AUC. Meanwhile, the non-sampling strategy has a statistically better performance than the two other sampling methods with regards to top-decile lift. There is no statistical difference with respect to EMP as shown by the Iman-Davenport test. So when you want to build a decision tree model for churn prediction, which sampling procedure to use depends upon 
the performance measure adopted. When AUC and EMP are used, RUS is recommended. However, the sampling solution is useless to improve top-decile lift.

Different from C4.5, most sampling techniques work well with SVM classifier across different measures. As demonstrated in Table 3, the non-sampling strategy stays at the bottom part of the ranking list. Holm's test shows that non-sampling is also statistically worse than the top ranking methods for both AUC and top-decile lift. For the EMP measure, there is no statistical difference between the different methods according to the Iman-Davenport test. We further investigate the results of each individual measure. ROS demonstrates its excellence in terms of AUC and top-decile lift, ranking first and second for the two measures, respectively. It is interesting to note that RUS is also the best performing sampling technique when using EMP. The results indicate that sampling solutions can be used for SVMs without any hesitation.

\subsection{Comparison of cost-sensitive learning}

Table 4: Experimental performance of cost-sensitive techniques

\begin{tabular}{lccccccc}
\hline \multirow{2}{*}{ Sampling } & \multicolumn{3}{c}{ C4.5 } & & \multicolumn{3}{c}{ SVM } \\
\cline { 2 - 3 } \cline { 5 - 7 } & AUC & Top-decile lift & EMP & & AUC & Top-decile lift & EMP \\
\hline None & 0.6557 & 4.5330 & 1.0359 & & 0.6756 & 2.7039 & 0.7233 \\
Cost-sensitive & 0.6780 & 5.2925 & 1.0364 & & 0.7255 & 3.3549 & 0.8864 \\
\hline Wilcoxon test & $p=0.4235$ & $p=0.4235$ & $p=0.959$ & & $p=0.0076$ & $p=0.0033$ & $p=0.1549$ \\
\hline
\end{tabular}

This subsection discusses the results of cost-sensitive solutions. Table 4 shows the average performance of costsensitive solutions in comparison to using no sampling method and baseline classifiers that assume equal misclassification costs ("None"). As before, each entry in the table presents the mean value of the three evaluation metrics and the last row offers the $p$-values of the Wilcoxon signed-rank test. From the table, we can see that the cost-sensitive solutions definitely improve the results over the two baseline classifiers across the three measures, but the degrees of improvement vary. For C4.5 decision tree, cost-sensitive learning does not have a significant increase for all the three measures according to the Wilcoxon signed-rank test. On the other hand, cost-sensitive SVM gives a statistically significant increase for both AUC and top-decile lift. However, there is still no significant difference for the EMP measure as the cost-sensitive tree. In summary, cost-sensitive techniques are useful for SVM and C4.5 to deal with class imbalance in churn prediction when the misclassification costs are known.

\subsection{Comparison of ensemble solution}

In this subsection, we will discuss the results of ensemble solutions. As mentioned in section 3, there are mainly four types of ensemble solutions: bagging-based, boosting-based, random-forest-based and hybrid ensemble, so that we will analyze the experimental results of each group, separately. The inter-group comparison can be found in the 
global comparison of the next subsection. In order to validate wether the solutions are beneficial to deal with class imbalance, the original Bagging, AdaBoost and random forest techniques are also used to provide contrast. Table 5 to Table 8 show the experimental results in each group, where each entry in these tables gives the mean value as well as mean rank of each metric over all the eleven data sets. The last row provides the $p$-values of Iman-Davenport test or the Wilcoxon signed-rank test. The methods are arranged according to their mean ranks in an ascending order and the ranks that are significantly worse than the best one are underlined based on Holm's post-hoc test.

1. Bagging-based ensemble. As Table 5 shows, all bagging-based solutions improve AUC performance over the Bagging method. However, only UnderBagging has a slight improvement over Bagging for top-decile lift and their ranks are the same. Meanwhile, no improvement can be found for any bagging-based solutions in terms of EMP. UnderBagging has the best performance in terms of AUC and it is significantly better than SMOTEBagging, OverBagging and Bagging methods according to the Holm's test. There is no statistical difference among the five methods for top-decile lift based on the Iman-Davenport test. The priority of Bagging with respect to EMP is significant except for the comparison with UnderBagging. It can also be found that the integration of undersampling with bagging is more efficient than that with oversampling for all the three measures. UnderBagging and RBBagging consistently demonstrate better ranks than OverBagging and SMOTEBagging. They should be considered when AUC and top-decile lift are used while no solutions can contribute to address the class imbalance in terms of EMP.

Table 5: Experimental performance of bagging-based methods

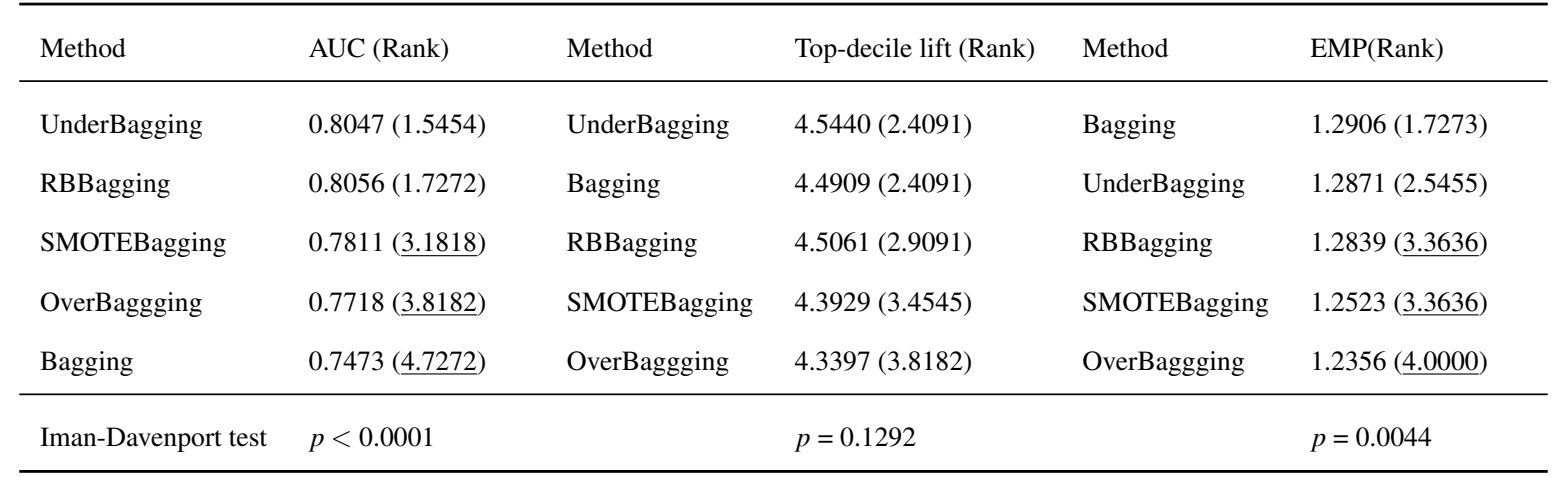

2. Boosting-based ensemble. Table 6 summarizes the experimental results of boosting-based solutions. As can be seen, the effectiveness of boosting-based techniques is ambiguous. In comparison with AdaBoost, AdaC2 has the worst performance for all the three metrics. RUSBoost takes the first place for both AUC and top-decile lift and it is worse than AdaBoost for EMP. SMOTEBoost only presents slightly better mean EMP values than AdaBoost but their mean ranks are the same. According to Holm's test, RUSBoost is significantly better than the other three methods with respect to AUC while it is only significantly better than AdaC2 in terms of top-decile lift. For EMP, SMOTEBoost and Adaboost are the top two methods, but there is no significant difference among the four methods according to the Iman-Davenport test. All the results show that in churn prediction, cost-sensitive ensembles are not a good option to cope with class imbalance. Moreover, RUSBoost should be considered for AUC and the top-decile measure while 
SMOTEBoost is recommenced for EMP among the boosting-based ensemble solutions.

Table 6: Experimental performance of boosting-based methods

\begin{tabular}{llllll}
\hline Method & AUC (Rank) & Method & Top-decile lift (Rank) & Method & EMP(Rank) \\
\hline RUSBoost & $0.7808(\mathbf{1 . 5 4 5 5})$ & RUSBoost & $4.2400(\mathbf{1 . 6 3 6 4})$ & SMOTEBoost & $1.2004(2.3636)$ \\
AdaBoost & $0.7610(2.5454)$ & AdaBoost & $4.1327(2.3636)$ & AdaBoost & $1.1992(2.3636)$ \\
SMOTEBoost & $0.7647(2.6364)$ & SMOTEBoost & $4.1689(2.4545)$ & RUSBoost & $1.1726(2.4545)$ \\
AdaC2 & $0.7523(\underline{3.2727})$ & AdaC2 & $3.9360(\underline{3.5454})$ & AdaC2 & $1.0771(2.8182)$ \\
\hline Iman-Davenport test & $p=0.0111$ & & $p=0.0027$ & & $p=0.8329$ \\
\hline
\end{tabular}

3. Random-forest-based ensemble. After careful examination, we can get some insights from Table 7. As for the boosting-based group, the effectiveness here heavily depends on the performance measure. Both weighted random forest (WRF) and balance random forest (BRF) show improvements over the original random forest for AUC but only the latter (BRF) increases the top-decile lift. At the same time, they are still worse than the original random forest algorithm in terms of EMP. According to Holm's test, BRF is significantly better than WRF and RF in terms of AUC and (original) random forest is statistically better than BRF for EMP based on Holm's test. BRF also ranks first in terms of top-decile lift but the difference is not significant among the three methods based on the Iman-Davenport test. The results advocate balanced random forests in terms of AUC and top-decile lift while no random-forest-based technique works well to address the class imbalance issue with regard to EMP measure.

Table 7: Experimental performance of random-forest-based methods

\begin{tabular}{llllll}
\hline \multicolumn{7}{l}{ Table 7: Experimental performance of random-forest-based methods } \\
\hline BRF & AUC(Rank) & Method & Top-decile lift (Rank) & Method & EMP(Rank) \\
WRF & $0.7952(1.2727)$ & BRF & $4.2890(1.7273)$ & RF & $1.2376(1.6364)$ \\
RF & $0.7671(\underline{2.3636})$ & RF & $4.2621(1.9091)$ & BRF & $1.1929(1.7273)$ \\
\hline \multirow{2}{*}{ Iman-Davenport test } & $p=0.0064$ & WRF & $4.0250(2.3636)$ & WRF & $1.1123(\underline{2.6364})$ \\
\hline
\end{tabular}

4. Hybrid ensemble. As table 8 shows, EasyEnsemble is better than BalanceCascade across three measures. Since there are only two methods, we used the Wilcoxon signed-rank test. According to this test, the superiority of EasyEnsemble is significant for both AUC and top-decile lift but not for the EMP measure.

\subsection{Global comparison}

In this subsection, a global comparison is conducted to find well-performing solutions for each evaluation metric. To make the analysis more concise, we focus our discussion on only two measures: AUC (general) and EMP (domain specific). To achieve this goal, we pick the representative methods which obtain better average ranks with respect 
Table 8: Experimental performance of hybrid ensemble methods

\begin{tabular}{lccc}
\hline Method & AUC(Rank) & Top-decile lift (Rank) & EMP(Rank) \\
\hline BalanceCascade & 0.7675 & 4.1564 & 1.1851 \\
EasyEnsemble & 0.7900 & 4.2973 & 1.1947 \\
\hline Wilcoxon test & $p=0.0076$ & $p=0.0409$ & $p=0.2477$ \\
\hline
\end{tabular}

to the two different measures from each group for a given measure. More specifically, from the sampling solutions, we select the best sampling methods for each baseline classifier. Since the two cost-sensitive learning solutions can improve over the baseline classifiers, they stand out from the baseline classifiers. Among the ensemble solutions, one method will be retained from the bagging-based, boosting-based, random forest-based and hybrid ensemble group, respectively. In total, eight representative methods are chosen to perform the global comparison for each measure. Table 9 shows the summary of the global comparison, where the second and the sixth column give the mean ranks over the eleven data sets. By using the Iman-Davenport test, we get two $p$-values that are smaller than 0.0001 for both measures, which help us to reject the null hypothesis that all representative methods have equivalent performance. Consequently, a Holm's test is performed and the third and seventh column in Table 9 give the $p$-values.

In general, we can see from Table 9 that ensemble solutions dominate the results. The four representative ensemble methods are suited at the top part of the table, especially the bagging-based methods. Another observation is that sampling solutions seem to be more effective than cost-sensitive solutions. For both SVM and C4.5, the sampling treatment always obtains a better ranking than the corresponding cost-sensitive versions with respect to both AUC and EMP measure. If we go one step further, we can see that the representative methods demonstrate some different behavior in terms of AUC and EMP. When AUC is considered, UnderBagging has the best value and it performs statistically better than all the non-ensemble techniques. When EMP is used, Bagging and random forest, two ensemble methods without considering class imbalance, have the best ranks. To summarize, the ensemble solutions are the best performing techniques in dealing with class imbalance, but they do not contribute to improve the profit-based measures.

\subsection{Summary and discussion}

From the above experimental analysis, we can summarize the main experiment findings and try to answer the research questions as follows:

RQ1 Do different evaluation metrics have similar influence on the performance of different solutions?

A: The evaluation metrics have a large impact on the (measured, ranked) performance of different techniques. Using profit-based measures leads to significantly different performance ranks in contrast with both top-decile lift and AUC, not only in intra-family comparison but also in the global comparison. This suggests that previous 
Table 9: Experimental performance of global comparison

\begin{tabular}{|c|c|c|c|c|c|c|c|}
\hline \multirow{2}{*}{ Method } & \multicolumn{3}{|c|}{ AUC } & \multirow{2}{*}{ Method } & \multicolumn{3}{|c|}{ EMP } \\
\hline & Rank & PV(Holm’s) & Action & & Rank & PV(Holm’s) & Action \\
\hline UnderBagging & 1.3636 & - & - & Bagging & 1.9091 & - & - \\
\hline $\mathrm{BRF}$ & 2.5455 & 0.2578 & Not Reject & Random forest & 3.5455 & 0.1172 & Not Reject \\
\hline EasyEnsemble & 2.5455 & 0.2578 & Not Reject & EasyEnsemble & 4.0000 & 0.0453 & Reject \\
\hline RUSBoost & 4.2727 & 0.0053 & Not Reject & SMOTEBoost & 4.4545 & 0.0148 & Reject \\
\hline ROS+SVM & 5.2727 & 0.0002 & Reject & RUS+SVM & 4.7273 & 0.0070 & Reject \\
\hline CS-SVM & 6.0909 & $<0.0001$ & Reject & CS-SVM & 4.8182 & 0.0053 & Reject \\
\hline $\mathrm{RUS}+\mathrm{C} 4.5$ & 6.5455 & $<0.0001$ & Reject & $\mathrm{RUS}+\mathrm{C} 4.5$ & 6.1364 & $<0.0001$ & Reject \\
\hline CS-Tree & 7.3636 & $<0.0001$ & Reject & CSTree & 6.4091 & $<0.0001$ & Reject \\
\hline
\end{tabular}

conclusions based on the general AUC measure should be carefully validated in a churn prediction context when profit is of concern.

RQ2 Does each solution truly improve the performance in comparison with the original technique without considering class imbalance?

A: As shown in the intra-family comparisons, we can see that different types of solutions have different behaviors in comparison with their counterpart without considering class imbalance. The specific behavior is listed as follows:

- Sampling methods do not grantee the improvement and their performance depends on the classifier. For C4.5 decision tree models, the influence depends on the evaluation measure: most sampling methods can improve AUC and EMP, but none can improve the top-decile measure. At the same time, SVM can gain benefits from resampling for all the three measures. In general, more complex sampling methods do not demonstrate any superior performance compared to simple random sampling methods such as RUS and ROS.

- Cost-sensitive learning can improve over baseline classifiers. The improvement is more obvious for costsensitive SVM models than cost-sensitive C4.5.

- With respect to ensemble solutions, most techniques integrated with sampling methods increase the performance in terms of AUC and some of them improve top-decile lift. However, ensemble methods embedding cost-sensitive learning decay the performance of these two measures. No ensemble solution has significant better EMP values than their counterpart without considering class imbalance.

RQ3 What is the best technique given an evaluation measure?

A: From the perspective of a global comparison, ensemble-based methods are still the most promising methods. 
For AUC, the combination of Bagging with random undersampling (UnderBagging, BRF, EasyEnsemble and RUSBoost) shows its superiority, especially UnderBagging. However, while techniques designed for imbalanced data sets do not show any superiority in terms of EMP, the original Bagging and Random forest methods beat other methods based on this measure. The reason behind this needs future research. Furthermore there is still room to develop algorithms that can deal with class imbalance and optimize for a profit-based evaluation.

\section{Conclusions}

Customer relationship management is an indispensable part of all business, in which churn prediction is the key step. Class imbalance is intrinsic to data sets in churn prediction. Dealing with this issue and identifying the minority churner group from a large number of non-churners is the core of successful churn modeling. In our study, we present a comprehensive comparison of state-of-the art techniques for dealing with class imbalance. A recently developed profit-based measure EMP is used to evaluate the results from the perspective of costs and benefits. The experimental results show that different types of solutions demonstrate different behaviors. An in-depth exploration of the reaction patterns to different measures is carefully provided. It is also found that ensemble methods are the most prominent methods and one interesting finding is that the original Bagging and random forest learning algorithm without considering class imbalance get the best results with respect to the profit-based measure. Our research provides some guidance to choose suitable methods to deal with class imbalance in both the academic community as well as practice. In the future, we plan to develop some new ensemble techniques to optimize the profit-based measure in the context of churn prediction with class imbalance.

\section{Acknowledgment}

This work is supported by the National Natural Science Foundation of China (Grant No. 71401115) and the MOE (Ministry of Education in China) Youth Project of Humanities and Social Sciences (Grant No. 13YJC630249). Bing Zhu is supported by the postdoctoral fellowship from the China Scholarship Council (CSC).

\section{Appendix}

See Table 10, Table11 and Table 12

\section{Reference}

[1] O. Ali, U. Ariturk, Dynamic churn prediction framework with more effective use of rare event data: The case of private banking, Expert Systems with Applications 41(17) (2014) 7889-7903.

[2] B. Baesens, Analytics in a Big Data World, John Wiley and Sons, 2014.

[3] S. Barua, M. Islam, X. Yao, MWMOTE-majority weighted minority oversampling technique for imbalanced data set learning, IEEE Transactions on Knowledge and Data Engineering 26(2) (2014) 405-425. 
[4] G.E. Batista, R.C. Prati, M.C.Monard, A study of the behavior of several methods for balancing machine learning training data, ACM Sigkdd Explorations Newsletter 6(1) (2004) 20-29.

[5] C.B. Bhattacharya, When customers are members: Customer retention in paid membership contexts, Journal of the Academy of Marketing Science, 26(1) (1998) 31-44.

[6] U. Bhowan, M. Johnston, M. Zhang, X. Yao, Reusing genetic programming for ensemble selection in classification of unbalanced data, IEEE Transactions on Evolutionary Computation 18(6) (2014) 893-908.

[7] A.P. Bradley, The use of the area under the roc curve in the evaluation of machine learning algorithms, Pattern Recognition 30(7) (1997) 1145-1159.

[8] J. Burez, D. Van den Poel, D, CRM at a pay-TV company: using analytical models to reduce customer attrition by targeted marketing for subscription services, Expert Systems with Applications 32 (2007) 277-288.

[9] J. Burez, D. Van den Poel, Handling class imbalance in customer churn prediction, Expert Systems with Applications 36(3, Part 1) (2009) 4626-4636.

[10] N.V. Chawla, K.W. Bowyer, L.O. Hall, W.P. Kegelmeyer, SMOTE: Syntheticminority over-sampling technique, Journal of Artificial Intelligence Research 16(3) (2002) 321-357.

[11] N. Chawla, A. Lazarevic, L. Hall, K.W. Bowyer, SMOTEBoost: Improving prediction of the minority class in boosting, in: Proceeding of PKDD 2003, pp. 107-119.

[12] Z. Chen, Z. Fan, M. Sun, A hierarchical multiple kernel support vector machine for customer churn prediction using longitudinal behavioral data, European Journal of Operational Research 223(2) (2012) 461-472.

[13] C. Chen, A. Liaw, L. Breiman, Using random forests to learn imbalanced data, Technical Report, Statistics Department of University of California at Berkeley, 2004.

[14] M. Colgate, P. Danaher, Implementing a customer relationship strategy: The asymmetric impact of poor versus excellent execution, Journal of the Academy of Marketing Science 28(3) (2000) 375-387.

[15] K. Coussement, K. De Bock, Customer churn prediction in the online gambling industry: The beneficial effect of ensemble learning, Journal of Business Research 66(9) (2014) 1629-1636.

[16] K. Coussement, D. Van den Poel, D. (2008). Churn prediction in subscription services: An application of support vector machines while comparing two parameterselection techniques, Expert Systems with Applications 34(1) (2008) 313C327.

[17] K. Coussement, S. Lessmann, G. Verstraeten, A comparative analysis of data preparation algorithms for customer churn prediction: A case study in the telecommunication industry,Decision Support Systems, 2016, online first.

[18] J. Demšar, Statistical comparisons of classifiers over multiple data sets, Journal of Machine Learning Research 7 (2006) 1-30.

[19] S. Ertekin, J. Huang, L. Bottou, C.L. Giles, Learning on the border: Active learning in imbalanced data classification, in: Proceedings of the sixteenth ACM conference on Conference on information and knowledge management, ACM, 2007, pp.127-136.

[20] V. García, V., J. S. Sànchez, R. Mollineda, On the effectiveness of preprocessing methods when dealing with different levels of class imbalance, Knowledge-Based Systems 25(1) (2012) 13-21.

[21] S. García, F. Herrera, Evolutionary undersampling for classification with imbalanced datasets: Proposals and taxonomy, Evoluionary Computation 17(3) (2009) 275-306.

[22] J. Ganesh, M. Arnold, K. Reynolds, Understanding the customer base of service providers: An examination of the differences between switchers and stayers, Journal of Marketing 64(3) (2000) 65-87.

[23] M. Galar, A. Fernández, E. Barrenechea, H. Bustince, F. Herrera, Ordering-based pruning for improving the performance of ensembles of classifiers in the framework of imbalanced datasets, Information Sciences 354 (2016) 178-196.

[24] M. Galar, A. Fernández, E. Barrenechea, H. Bustince, F. Herrera, A review on ensembles for the class imbalance problem: bagging-, boosting-, and hybrid-based approaches, IEEE Transactions on Systems, Man, and Cybernetics, Part C: Applications and Reviews 41(4) (2012) 463-484.

[25] N. Gordini, V. Veglio, Customers churn prediction and marketing retention strategies. An application of support vector machines based on 
the AUC parameter-selection technique in B2B e-commerce industry Industrial Marketing Management, 2016, online first.

[26] J. Hadden, A. Tiwari, R. Roy, D. Ruta, Computer assisted customer churn management: State-of-the-art and future trends, Computers \& Operations Research 34(10) (2007) 2902-2917.

[27] H. He, Y. Bai, E.A. Garcia, S. Li, ADASYN: Adaptive synthetic sampling approach for imbalanced learning, in: Proc. IEEE International Joint Conference on Neural Networks, 2008, pp. 1322-1328.

[28] H. He, E.A. Garcia, Learning from imbalanced data, IEEE Transactions on Knowledge and Data Engineering 21(9) (2009) 1263-1284.

[29] S. Hido, H. Kashima, Y. Takahashi, Roughly balanced bagging for imbalanced data, Statistical Analysis and Data Mining 2(5-6) (2009) 412-426.

[30] S. Holm, A simple sequentially rejective multiple test procedure, Scandinavian journal of statistics 6 (1979) 65-70.

[31] S. Hung, D. Yen, H. Wang, Applying data mining to telecom churn management, Expert Syst Appl 31(3) (2006) $515 C 524$.

[32] B. Huang, B. Buckley, T. Kechadi, Multi-objective feature selection by using NSGA-II for customer churn prediction in telecommunications, Expert Systems with Applications 37(5) (2010) 3638-3646.

[33] A. Idris, M. Rizwan, A. Khan. Churn prediction in telecom using Random Forest and PSO based data balancing in combination with various feature selection strategies, Computers \& Electrical Engineering 38(6) (2012) 1808-1819.

[34] L. Iman James, J.M. Davenport, Approximations of the critical region of the fbietkan statistic, Communications in Statistics Theory and Methods 9(6) (1980) 571-595.

[35] A. Jahromi, S. Stakhovych, M. Ewing, Managing B2B customer churn, retention and profitability, Industrial Marketing Management 43(7) 2014 1258-1268.

[36] Z. Jamal, R. Bucklin, Improving the diagnosis and prediction of customer churn: A heterogeneous hazard modeling approach, Journal of Interactive Marketing 20(3-4) (2006) 16-29.

[37] A. Keramati, R. Jafari-Marandi, M. Aliannejadi, I. Ahmadian, M. Mozaffari, U. Abbasi, Improved churn prediction in telecommunication industry using data mining techniques, Applied Soft Computing 24 (2014) 994-1012.

[38] M. Kubat, S. Matwin, Addressing the curse of imbalanced training sets: one-sided selection, in: Proceeding of International Conference on Machine Learning, 1997, pp. 179-186.

[39] T. M. Khoshgoftaar, J. Van Hulse, A. Napolitano, Comparing boosting and bagging techniques with noisy and imbalanced data, IEEE Transactions on Systems, Man and Cybernetics, Part A: Systems and Humans 99 (2010) 1-17.

[40] A. Lemmens, C. Croux, Bagging and boosting classification trees to predict churn, Journal of Marketing Research (JMR) 43 (2006) $276-286$.

[41] E. Lima, C. Mues, B.Baesens, Domain knowledge integration in data mining using decision tables: case studies in churn prediction, Journal of the Operational Research Society 8 (2009) 1096-1106.

[42] X, Liu, J. Wu, Z. Zhou, Exploratory undersampling for class-imbalance learning, IEEE Transactions on Systems, Man, and Cybernetics, Part B: Cybernetics 39(2) (2009) 539-550.

[43] V. López, A. Fernández, J. G. Moreno-Torres, F. Herrera, Analysis of preprocessing vs. cost-sensitive learning for imbalanced classification. Open problems on intrinsic data characteristics, Expert Systems with Applications 39(7) (2012) 6585-6608.

[44] V. López, A. Fernández, S. García, V. Palade, F. Herrera, An insight into classification with imbalanced data: Empirical results and current trends on using data intrinsic characteristics, Information Sciences 250 (2013) 113-141.

[45] S. Neslin, S. Gupta, W. Kamakura, J. Lu, C. Mason, Detection defection: Measuring and understanding the predictive accuracy of customer churn models, Journal of Marketing Research 43(2) (2006) 204-211.

[46] H. Lee, Y. Lee, H. Cho, K. Im, Y. Kim. Mining churning behaviors and developing retention strategies based on a partial least squares (PLS) model, Decision Support Systems 52(1) (2011) 207-216.

[47] T. Raeder, G. Forman, N.V. Chawla, Learning from imbalanced data: evaluation matters, Data Mining: Foundations and Intelligent Paradigms, Springer (2012) 315-331.

[48] A. Raskutti, A. Kowalczyk, Extreme rebalancing for SVMs: A case Study, ACM SIGKDD Explorations Newsletter 6(1) (2004) 60-69.

[49] C. SeiffertT, M. Khoshgoftaar, J. Van Hulse, A. Folleco, An empirical study of the classification performance of learners on imbalanced and 
noisy software quality data, Information Sciences 259 (2014) 571-595.

[50] C. Seiffert, T. M. Khoshgoftaar, J. Van Hulse, A. Napolitan, A comparative study of data sampling and cost sensitive learning, in: Proceedings of IEEE International Conference on Data Mining Workshops, 2008, pp. 46-54.

[51] C. Seiffert, T. M. Khoshgoftaar, J. Van Hulse, RUSBoost: A hybrid approach to alleviating class imbalance, IEEE Transactions on Systems, Man and Cybernetics, Part A: Systems and Humans 40(1) (2010) 185-197.

[52] H.J. Shin, D.H. Eom, S.S. Kim, One-Class Support Vector Machines-An Application in Machine Fault Detection and Classification, Computers \& Industrial Engeering, 48(2) (2005) 395-408.

[53] Y. Sun, M. S. Kamel, A. K. Wong, Y. Wang, Cost-sensitive boosting for classification of imbalanced data, Pattern Recognition, 40(12) 2007 3358-3378.

[54] Y. Sun, A.K.Wong, M.S. Kamel, Classification of imbalanced data: A review, International Journal of Pattern Recognition and Artificial Intelligence 23(4) 2009 687-719.

[55] Z.Sun, Q. Song, X. Zhu, H. Sun, B. X, Y. Zhou, A novel ensemble method for classifying imbalanced data, Pattern Recognition, 48(5) 2015 1623-1637.

[56] C. Tsai, Y. Lu, Customer churn prediction by hybrid neural networks.” Expert Systems with Applications 36(10)(2009 12547-12553.

[57] K.M. Ting, An instance-weighting method to induce cost-sensitive trees, IEEE Transactions on Knowledge and Data Engineering 14(3) (2002) 659-665.

[58] Q. Yang, X. Wu, 10 challenging problems in data mining research, International Journal of Information Technology Decision Making 5(4) (2006) 597-604.

[59] P. Yang, L. Xu, B.B. Zhou, Z. Zhang, A.Y. Zomaya, A particle swarm based hybrid system for imbalanced medical data sampling, BMC Genomics, 10(Suppl 3)(2009) S34.

[60] S.J. Yen, Y.S. Lee, Cluster-based under-sampling approaches for imbalanced data distributions, Expert Systems with Applications 36(3) (2009) 5718-5727.

[61] V. Zeithaml, L. Berry, A. Parasuraman, The behavioural consequences of service quality, Journal of Marketing 60(2) (1996) 31-46.

[62] X. Zhang, J. Zhu, S. Xu, Y. Wan, Predicting customer churn through interpersonal influence, Knowledge-Based System 28 (2012) 97-104.

[63] B. Zhu, B. Baesens, A. Backiel, S. vanden Broucke, Benchmarking sampling techniques for imbalance learning in churn prediction, Journal of the Operational Research Society (Accepted)

[64] Z. Zhou, X. Liu, Training cost-sensitive neural networks with methods addressing the class imbalance problem, IEEE Transactions on Knowledge and Data Engineering 18(1) (2006) 63-77.

[65] J. Van Hulse, T. M. Khoshgoftaar, A. Napolitano, Experimental perspectives on learning from imbalanced data, in : Proceedings of the 24th international conference on Machine learning, 2007, pp. 935-942.

[66] W. Verbeke, D. Martens, C. Mues, B. Baesens, Building comprehensible customer churn prediction models with advanced rule induction techniques, Expert Systems with Applications 38(3) (2011) 2354-2364.

[67] W. Verbeke, K. Dejaeger, D. Martens, J. Hur, B. Baesens, New insights into churn prediction in the telecommunication sector: A profit driven data mining approach, European Journal of Operational Research 218(1) (2012) 211-229.

[68] W. Verbeke, D. Martens, B. Baesens, Social network analysis for customer churn prediction, Applied Soft Computing 14 (2014) $431-446$.

[69] T. Verbraken, W. Verbeke, B. Baesens, A novel profit maximizing metric for measuring classification performance of customer churn prediction models, IEEE Transactions on Knowledge and Data Engineering 25(5) (2013) 961-973.

[70] K. Veropoulos, C. Campbell, N. Cristianini, Controlling the sensitivity of support vector machines, in: Proceedings of the international joint conference on AI, 1999, pp. 55-60.

[71] S. Wang, X. Yao, Diversity analysis on imbalanced data sets by using ensemble models, in : IEEE Symposium on Computational Intelligence, 2009, pp. 324-331.

[72] G. Weiss, Mining with rarity: a unifying framework, ACM Sigkdd Explorations Newsletter 6(1) (2004) 7-19.

[73] G. Weiss, K. McCarthy, B. Zabar, Cost-sensitive learning vs. sampling: which is best for handling unbalanced classes with unequal error 
costs, in: Proceedings of the International Conference on Data Mining, 2007, pp. 35-41.

[74] F. Wilcoxon, Individual comparisons by ranking methods, Biometrics bulletin 1(6) (1945) 80-83.

[75] J. Xiao, L. Xie, C. He, X. Jiang, Dynamic classifier ensemble model for customer classification with imbalanced distribution, Expert Systems with Applications 39 (2012) 3668-3675.

[76] Y. Xie, X. Li, E. Ngai, M. Ying, Customer churn prediction using improved balanced random forests, Expert Systems with Applications, 36 (3, Part 1) (2009) 5445-5449. 
Table 10: Detailed experimental results (AUC)

\begin{tabular}{|c|c|c|c|c|c|c|c|c|c|c|c|}
\hline method & Chile & Duke1 & Duke2 & KDDcup & $\mathrm{K} 1$ & $\mathrm{~K} 2$ & K3 & K4 & K5 & Tele1 & UCI \\
\hline $\mathrm{C} 4.5$ & 0.6114 & 0.5552 & 0.5921 & 0.5676 & 0.5000 & 0.5574 & 0.7421 & 0.9599 & 0.6312 & 0.6368 & 0.8591 \\
\hline C4.5_ADASYN & 0.6518 & 0.5160 & 0.5388 & 0.5989 & 0.6554 & 0.6377 & 0.7466 & 0.9558 & 0.7492 & 0.7055 & 0.8333 \\
\hline C4.5+CLUS & 0.6517 & 0.5192 & 0.5394 & 0.6230 & 0.7750 & 0.7528 & 0.8665 & 0.9495 & 0.7484 & 0.7001 & 0.8552 \\
\hline C4.5+MWMOTE & 0.6357 & 0.5088 & 0.5203 & 0.5726 & 0.5675 & 0.6226 & 0.7414 & 0.9470 & 0.7089 & 0.7173 & 0.8447 \\
\hline C4.5+ROS & 0.6346 & 0.5027 & 0.5084 & 0.5285 & 0.5838 & 0.5946 & 0.7074 & 0.9342 & 0.6480 & 0.6550 & 0.8133 \\
\hline C4.5+RUS & 0.6296 & 0.5374 & 0.5619 & 0.6296 & 0.7620 & 0.7780 & 0.8555 & 0.9621 & 0.7473 & 0.7334 & 0.8570 \\
\hline C4.5+SMOTE & 0.6564 & 0.5250 & 0.5388 & 0.6046 & 0.6264 & 0.6477 & 0.7417 & 0.9434 & 0.7560 & 0.7068 & 0.8529 \\
\hline C4.5+SMOTE-ENN & 0.6479 & 0.5226 & 0.5364 & 0.5860 & 0.6272 & 0.6672 & 0.8440 & 0.9244 & 0.7405 & 0.6867 & 0.8328 \\
\hline C4.5+SMOTE-TL & 0.6319 & 0.5181 & 0.5365 & 0.5968 & 0.6167 & 0.6296 & 0.7588 & 0.9491 & 0.7626 & 0.7346 & 0.8393 \\
\hline SVM & 0.6550 & 0.5289 & 0.5351 & 0.5733 & 0.6431 & 0.7786 & 0.8672 & 0.6529 & 0.7111 & 0.6014 & 0.8851 \\
\hline SVM+ADASYN & 0.6827 & 0.5506 & 0.5506 & 0.6236 & 0.6763 & 0.8142 & 0.9393 & 0.7480 & 0.7814 & 0.7361 & 0.8874 \\
\hline SVM+CLUS & 0.6953 & 0.5454 & 0.5402 & 0.6818 & 0.6716 & 0.8395 & 0.9241 & 0.6998 & 0.7416 & 0.7843 & 0.8676 \\
\hline SVM+MWMOTE & 0.6897 & 0.5536 & 0.5555 & 0.6366 & 0.6767 & 0.8169 & 0.9348 & 0.7458 & 0.7796 & 0.7277 & 0.8903 \\
\hline SVM+ROS & 0.6984 & 0.5471 & 0.5564 & 0.6379 & 0.7074 & 0.8317 & 0.9363 & 0.7314 & 0.7856 & 0.7380 & 0.8881 \\
\hline SVM+RUS & 0.7072 & 0.5494 & 0.5443 & 0.6632 & 0.6634 & 0.8438 & 0.9259 & 0.7037 & 0.7607 & 0.7583 & 0.8775 \\
\hline SVM+SMOTE & 0.6977 & 0.5523 & 0.5501 & 0.6268 & 0.7125 & 0.8072 & 0.9340 & 0.7209 & 0.7718 & 0.7374 & 0.8850 \\
\hline SVM+SMOTE-ENN & 0.7000 & 0.5730 & 0.5545 & 0.6474 & 0.6983 & 0.8213 & 0.9319 & 0.7400 & 0.7722 & 0.7484 & 0.8820 \\
\hline SVM+SMOTE-TL & 0.6928 & 0.5570 & 0.5492 & 0.6380 & 0.6980 & 0.8196 & 0.9296 & 0.7370 & 0.7759 & 0.7407 & 0.8885 \\
\hline CSSVM & 0.6978 & 0.5287 & 0.5418 & 0.6516 & 0.6945 & 0.8278 & 0.9316 & 0.6890 & 0.7735 & 0.7623 & 0.8823 \\
\hline CSTree & 0.6417 & 0.5054 & 0.5098 & 0.5593 & 0.5760 & 0.6117 & 0.9343 & 0.9364 & 0.6537 & 0.7004 & 0.8297 \\
\hline AdaBoost & 0.6993 & 0.5391 & 0.5538 & 0.6502 & 0.7329 & 0.8233 & 0.9215 & 0.9710 & 0.7714 & 0.7957 & 0.9126 \\
\hline AdaC2 & 0.6975 & 0.5348 & 0.5573 & 0.6306 & 0.7621 & 0.8145 & 0.8883 & 0.9643 & 0.7600 & 0.7757 & 0.8896 \\
\hline RUSBoost & 0.6931 & 0.5673 & 0.5788 & 0.6748 & 0.8115 & 0.8631 & 0.9284 & 0.9655 & 0.7979 & 0.8093 & 0.8995 \\
\hline SMOTEBoost & 0.7141 & 0.5311 & 0.5564 & 0.6508 & 0.7521 & 0.8534 & 0.9276 & 0.9628 & 0.7773 & 0.7752 & 0.9109 \\
\hline Bagging & 0.6834 & 0.5143 & 0.5158 & 0.6760 & 0.6454 & 0.8577 & 0.9302 & 0.9595 & 0.7369 & 0.7890 & 0.9116 \\
\hline OverBagging & 0.7214 & 0.5452 & 0.5615 & 0.6757 & 0.7457 & 0.8638 & 0.9140 & 0.9672 & 0.7878 & 0.7978 & 0.9095 \\
\hline SMOTEBagging & 0.7086 & 0.5607 & 0.5716 & 0.6817 & 0.7702 & 0.8707 & 0.9368 & 0.9599 & 0.8099 & 0.8099 & 0.9118 \\
\hline RBBagging & 0.7217 & 0.6005 & 0.6117 & 0.7179 & 0.8442 & 0.8800 & 0.9479 & 0.9651 & 0.8281 & 0.8284 & 0.9158 \\
\hline UnderBagging & 0.7140 & 0.6021 & 0.6147 & 0.7180 & 0.8316 & 0.8846 & 0.9444 & 0.9648 & 0.8290 & 0.8292 & 0.9194 \\
\hline Random forest & 0.6796 & 0.5409 & 0.5376 & 0.6700 & 0.7517 & 0.8386 & 0.9112 & 0.9712 & 0.7839 & 0.7745 & 0.9126 \\
\hline $\mathrm{BRF}$ & 0.7035 & 0.5768 & 0.6165 & 0.7097 & 0.8288 & 0.8810 & 0.9162 & 0.9662 & 0.8233 & 0.8190 & 0.9062 \\
\hline WRF & 0.7085 & 0.5325 & 0.5661 & 0.6473 & 0.7882 & 0.8566 & 0.8954 & 0.9618 & 0.7960 & 0.7841 & 0.9017 \\
\hline EasyEnsemble & 0.7080 & 0.5768 & 0.5831 & 0.6895 & 0.8170 & 0.8788 & 0.9378 & 0.9658 & 0.8080 & 0.8202 & 0.9047 \\
\hline BalanceCascade & 0.6944 & 0.5243 & 0.5498 & 0.6404 & 0.7898 & 0.8439 & 0.9315 & 0.9720 & 0.7742 & 0.7956 & 0.9060 \\
\hline
\end{tabular}


Table 11: Detailed experimental results (Top-decile lift)

\begin{tabular}{|c|c|c|c|c|c|c|c|c|c|c|c|}
\hline method & Chile & Duke1 & Duke2 & KDDcup & $\mathrm{K} 1$ & $\mathrm{~K} 2$ & K3 & K4 & K5 & Tele1 & UCI \\
\hline $\mathrm{C} 4.5$ & 5.7673 & 1.3499 & 1.5751 & 1.3222 & 4.7970 & 4.5144 & 5.4215 & 9.0581 & 6.0506 & 4.8807 & 5.1267 \\
\hline C4.5+ADASYN & 3.2579 & 1.1522 & 1.4752 & 2.0474 & 3.3356 & 3.8832 & 6.0018 & 8.6186 & 4.1551 & 3.5806 & 4.9009 \\
\hline C4.5+CLUS & 2.0252 & 1.0990 & 1.0581 & 1.7986 & 3.6223 & 3.6329 & 7.2286 & 8.8671 & 4.7056 & 2.3853 & 4.1943 \\
\hline C4.5+MWMOTE & 3.0943 & 1.1968 & 1.2286 & 1.9798 & 2.8555 & 4.0742 & 5.7520 & 8.8478 & 4.2405 & 3.6383 & 4.6185 \\
\hline $\mathrm{C} 4.5+\mathrm{ROS}$ & 4.4340 & 0.6547 & 0.7604 & 1.7330 & 5.9496 & 5.8972 & 6.5454 & 8.7715 & 4.1081 & 3.2765 & 4.6996 \\
\hline C4.5+RUS & 2.3899 & 1.2840 & 1.3753 & 2.3230 & 2.5668 & 3.7212 & 6.7070 & 9.1345 & 4.7021 & 3.1874 & 4.7557 \\
\hline C4.5+SMOTE & 3.2704 & 1.2797 & 1.3883 & 2.0641 & 3.4797 & 3.7654 & 6.0092 & 8.8479 & 4.2858 & 3.6907 & 4.5616 \\
\hline C4.5+SMOTE-ENN & 3.1321 & 1.2478 & 1.3492 & 2.1675 & 3.7436 & 3.9273 & 7.2653 & 8.8097 & 4.2945 & 3.5544 & 4.3710 \\
\hline C4.5+SMOTE-TL & 3.2579 & 1.2840 & 1.3275 & 2.0504 & 3.3594 & 4.0157 & 6.2736 & 8.8479 & 4.1847 & 3.7117 & 4.4843 \\
\hline SVM & 3.0377 & 1.1522 & 1.2721 & 1.3308 & 2.0638 & 3.5156 & 6.0606 & 2.2932 & 3.3032 & 1.2267 & 4.4875 \\
\hline SVM+ADASYN & 3.4151 & 1.3669 & 1.4589 & 2.1433 & 1.6318 & 4.1628 & 7.8530 & 2.6563 & 3.9460 & 3.6697 & 4.5862 \\
\hline SVM+CLUS & 3.4591 & 1.3265 & 1.2036 & 2.5218 & 2.3997 & 4.0010 & 7.8383 & 2.2169 & 3.0053 & 3.2870 & 4.1871 \\
\hline SVM+MWMOTE & 3.4151 & 1.4009 & 1.5045 & 2.2992 & 2.1357 & 4.0010 & 7.9192 & 2.5990 & 3.7980 & 3.6121 & 4.4947 \\
\hline SVM+ROS & 3.4465 & 1.3095 & 1.4361 & 2.3719 & 2.2558 & 4.0010 & 7.9265 & 2.4651 & 4.1203 & 3.7379 & 4.4873 \\
\hline SVM+RUS & 3.6038 & 1.3754 & 1.3286 & 2.4259 & 2.0397 & 4.3100 & 7.8457 & 2.1213 & 3.3049 & 3.7588 & 4.3073 \\
\hline SVM+SMOTE & 3.3396 & 1.2861 & 1.4383 & 2.3941 & 2.4958 & 4.1922 & 7.8751 & 2.2169 & 3.8502 & 3.6802 & 4.4553 \\
\hline SVM+SMOTEENN & 3.2453 & 1.5093 & 1.4926 & 2.5198 & 2.1599 & 4.1774 & 7.8090 & 2.5608 & 3.8624 & 3.6907 & 4.1870 \\
\hline SVM+SMOTETL & 3.3208 & 1.4243 & 1.4730 & 2.4592 & 2.1598 & 4.2952 & 7.8016 & 2.5226 & 3.8851 & 3.6855 & 4.5227 \\
\hline CSSVM & 3.6289 & 1.0948 & 1.3014 & 2.4739 & 2.2798 & 4.1482 & 7.8090 & 2.1593 & 3.9164 & 3.6645 & 4.4275 \\
\hline CSTree & 4.9120 & 1.0416 & 1.1265 & 1.8324 & 5.5418 & 8.6789 & 7.8604 & 9.5550 & 9.3729 & 4.1153 & 4.1801 \\
\hline AdaBoost & 3.7987 & 1.3477 & 1.4882 & 2.1453 & 3.0715 & 3.6038 & 7.2580 & 8.9244 & 4.4234 & 3.8375 & 5.5614 \\
\hline AdaC2 & 3.4591 & 1.1819 & 1.4763 & 1.9424 & 3.8155 & 3.5744 & 6.3103 & 8.9435 & 4.2858 & 3.5334 & 4.7736 \\
\hline RUSBoost & 3.4465 & 1.4051 & 1.6870 & 2.2614 & 4.1516 & 3.7068 & 7.2066 & 9.0200 & 4.6812 & 4.1311 & 4.9429 \\
\hline SMOTEBoost & 3.6730 & 1.2563 & 1.5111 & 2.1206 & 3.5996 & 3.6774 & 7.4490 & 8.8862 & 4.4948 & 3.7379 & 5.4520 \\
\hline Bagging & 3.7925 & 1.1947 & 1.2199 & 2.6848 & 3.7192 & 4.2658 & 8.2203 & 9.2109 & 5.1272 & 4.4771 & 5.4873 \\
\hline OverBagging & 3.8931 & 1.3988 & 1.5654 & 2.3305 & 3.4556 & 3.8392 & 7.8016 & 9.1154 & 4.7161 & 4.1625 & 5.4589 \\
\hline RBBagging & 3.7925 & 1.7559 & 1.7392 & 2.7645 & 4.2473 & 3.9127 & 7.9412 & 8.9817 & 4.9251 & 4.1048 & 5.4026 \\
\hline SMOTEBagging & 3.7736 & 1.4264 & 1.6186 & 2.3381 & 3.7195 & 4.2068 & 7.8530 & 8.9245 & 4.7980 & 4.2149 & 5.4484 \\
\hline UnderBagging & 3.9182 & 1.7516 & 1.7544 & 2.7671 & 4.3434 & 4.1039 & 7.8604 & 8.9817 & 5.0279 & 4.1048 & 5.3707 \\
\hline Random forest & 3.6541 & 1.2287 & 1.3210 & 2.2745 & 3.5036 & 3.7214 & 7.5739 & 8.9434 & 4.9426 & 4.1835 & 5.5366 \\
\hline $\mathrm{BRF}$ & 3.6478 & 1.3881 & 1.7522 & 2.5041 & 3.8633 & 4.0450 & 7.1625 & 8.8670 & 5.0279 & 3.8218 & 5.0988 \\
\hline WRF & 3.7170 & 1.1394 & 1.3492 & 1.6533 & 3.9595 & 4.2069 & 6.6409 & 8.7142 & 4.8171 & 3.0668 & 5.0102 \\
\hline EasyEnsemble & 3.6541 & 1.4732 & 1.7316 & 2.3643 & 3.8156 & 3.9569 & 7.5739 & 8.9627 & 4.6847 & 4.0472 & 5.0067 \\
\hline BalanceCascade & 3.5723 & 1.1245 & 1.3492 & 2.0721 & 3.6716 & 3.8392 & 7.3976 & 9.0009 & 4.4304 & 4.0682 & 5.1940 \\
\hline
\end{tabular}


Table 12: Detailed experimental results (EMP)

\begin{tabular}{|c|c|c|c|c|c|c|c|c|c|c|c|}
\hline method & Chile & Duke1 & Duke2 & KDDcup & $\mathrm{K} 1$ & $\mathrm{~K} 2$ & K3 & K4 & K5 & Tele1 & UCI \\
\hline $\mathrm{C} 4.5$ & 0.6893 & 0.0000 & 0.0000 & 0.0759 & 0.0328 & 0.1220 & 1.0435 & 2.2213 & 0.5480 & 1.0329 & 5.6294 \\
\hline C4.5+ADASYN & 0.5269 & 0.0000 & 0.0000 & 0.1694 & 0.1963 & 0.2932 & 1.0192 & 2.1745 & 0.4991 & 1.2296 & 5.4883 \\
\hline C4.5+CLUS & 0.2515 & 0.0000 & 0.0000 & 0.0788 & 0.1394 & 0.3280 & 1.0879 & 2.1562 & 0.4296 & 0.5813 & 5.7375 \\
\hline C4.5+MWMOTE & 0.4729 & 0.0000 & 0.0000 & 0.1384 & 0.1313 & 0.2296 & 0.9836 & 2.1838 & 0.4225 & 1.1812 & 5.5126 \\
\hline C4.5+ROS & 0.5833 & 0.0000 & 0.0000 & 0.0696 & 0.1039 & 0.2247 & 0.7694 & 2.2190 & 0.4443 & 1.0890 & 5.4509 \\
\hline C4.5+RUS & 0.1805 & 0.0000 & 0.0006 & 0.2621 & 0.2272 & 0.4075 & 1.1776 & 2.1345 & 0.4675 & 1.1598 & 5.6320 \\
\hline C4.5+SMOTE & 0.5643 & 0.0000 & 0.0000 & 0.1583 & 0.1985 & 0.3481 & 1.0446 & 2.1724 & 0.4768 & 1.1818 & 5.6119 \\
\hline C4.5+SMOTE-ENN & 0.4966 & 0.0000 & 0.0000 & 0.1750 & 0.1049 & 0.3339 & 1.2734 & 2.1617 & 0.4645 & 1.2259 & 5.5420 \\
\hline C4.5+SMOTE-TL & 0.4914 & 0.0000 & 0.0000 & 0.1786 & 0.1855 & 0.2341 & 1.1225 & 2.1628 & 0.4711 & 1.1841 & 5.7047 \\
\hline SVM & 0.7357 & 0.0006 & 0.0012 & 0.0947 & 0.0327 & 0.2985 & 0.9602 & 0.1005 & 0.1409 & 0.1704 & 5.4205 \\
\hline SVM+ADASYN & 0.6523 & 0.0000 & 0.0007 & 0.2458 & 0.0727 & 0.3906 & 1.4136 & 0.1498 & 0.2943 & 1.2742 & 5.2499 \\
\hline SVM+CLUS & 0.7241 & 0.0002 & 0.0006 & 0.2335 & 0.0894 & 0.3334 & 1.4786 & 0.0826 & 0.1571 & 1.1274 & 5.0207 \\
\hline SVM+MWMOTE & 0.6858 & 0.0004 & 0.0003 & 0.3123 & 0.0588 & 0.4174 & 1.4364 & 0.0624 & 0.3053 & 1.2689 & 5.2400 \\
\hline SVM+ROS & 0.7053 & 0.0007 & 0.0007 & 0.3019 & 0.1060 & 0.3698 & 1.3993 & 0.0936 & 0.3389 & 1.2455 & 5.2478 \\
\hline SVM+RUS & 0.6980 & 0.0009 & 0.0052 & 0.3098 & 0.0287 & 0.4337 & 1.4921 & 0.1071 & 0.1800 & 1.2870 & 5.0407 \\
\hline SVM+SMOTE & 0.6327 & 0.0004 & 0.0006 & 0.3155 & 0.1234 & 0.3708 & 1.4056 & 0.1310 & 0.2558 & 1.2407 & 5.3447 \\
\hline SVM+SMOTE-ENN & 0.5778 & 0.0000 & 0.0005 & 0.3441 & 0.0733 & 0.3803 & 1.4528 & 0.1339 & 0.2852 & 1.2813 & 5.3077 \\
\hline SVM+SMOTE-TL & 0.6558 & 0.0000 & 0.0011 & 0.3230 & 0.1057 & 0.4274 & 1.4310 & 0.1287 & 0.3113 & 1.2619 & 5.2094 \\
\hline CSSVM & 0.7301 & 0.0012 & 0.0037 & 0.3372 & 0.0379 & 0.4244 & 1.4474 & 0.0636 & 0.2435 & 1.2400 & 5.2209 \\
\hline CSTree & 0.6584 & 0.0000 & 0.0000 & 0.0855 & 0.0863 & 0.1788 & 1.4556 & 2.1407 & 0.4287 & 1.2466 & 5.1198 \\
\hline AdaBoost & 0.9058 & 0.0004 & 0.0019 & 0.2191 & 0.1656 & 0.2687 & 1.2387 & 2.2859 & 0.5450 & 1.3578 & 6.2028 \\
\hline AdaC2 & 0.7266 & 0.0016 & 0.0029 & 0.1385 & 0.2166 & 0.2727 & 0.9751 & 2.3295 & 0.5161 & 1.1508 & 5.5172 \\
\hline RUSBoost & 0.6833 & 0.0002 & 0.0018 & 0.2580 & 0.3252 & 0.3723 & 1.1767 & 2.2004 & 0.5578 & 1.4773 & 5.8458 \\
\hline SMOTEBoost & 0.8574 & 0.0003 & 0.0021 & 0.2001 & 0.2132 & 0.3585 & 1.2703 & 2.2503 & 0.5222 & 1.2680 & 6.2616 \\
\hline Bagging & 0.8749 & 0.0009 & 0.0063 & 0.4295 & 0.1902 & 0.4695 & 1.4552 & 2.2814 & 0.6525 & 1.5778 & 6.2587 \\
\hline OverBagging & 0.9115 & 0.0012 & 0.0020 & 0.2827 & 0.1345 & 0.4297 & 1.3570 & 2.2498 & 0.5786 & 1.4740 & 6.1710 \\
\hline RBBagging & 0.8199 & 0.0001 & 0.0010 & 0.4666 & 0.2881 & 0.4404 & 1.4496 & 2.2581 & 0.6321 & 1.5254 & 6.2419 \\
\hline SMOTEBagging & 0.8706 & 0.0003 & 0.0044 & 0.2851 & 0.2306 & 0.4072 & 1.3867 & 2.2769 & 0.5805 & 1.5442 & 6.1891 \\
\hline UnderBagging & 0.8429 & 0.0004 & 0.0020 & 0.4684 & 0.2484 & 0.4635 & 1.4510 & 2.2587 & 0.6267 & 1.5325 & 6.2632 \\
\hline $\mathrm{RF}$ & 0.8534 & 0.0002 & 0.0038 & 0.3227 & 0.2179 & 0.2995 & 1.3235 & 2.2328 & 0.6188 & 1.4450 & 6.2960 \\
\hline $\mathrm{BRF}$ & 0.7034 & 0.0010 & 0.0016 & 0.3798 & 0.2667 & 0.4402 & 1.1396 & 2.0986 & 0.5979 & 1.3831 & 6.1104 \\
\hline WRF & 0.7030 & 0.0007 & 0.0003 & 0.0685 & 0.2521 & 0.4073 & 1.0016 & 2.1471 & 0.5721 & 1.0969 & 5.9859 \\
\hline EasyEnsemble & 0.7261 & 0.0004 & 0.0012 & 0.2974 & 0.2691 & 0.4075 & 1.3084 & 2.1494 & 0.5532 & 1.4750 & 5.9547 \\
\hline BalanceCascade & 0.7143 & 0.0004 & 0.0012 & 0.1908 & 0.2226 & 0.3506 & 1.2659 & 2.2757 & 0.5472 & 1.4430 & 6.0242 \\
\hline
\end{tabular}

